Our Nature (2006)4:91-95

\title{
Diversity of Wetland Birds around the Koshi Barrage Area
}

\author{
D. T. Chhetry \\ Department of Zoology, Post Graduate Campus, Biratnagar.
}

Received: 21.10.2006; Accepted: 23.12.2006

\begin{abstract}
Koshi Barrage area comprises of a large reservoir, marshlands channels, floodplain, reed beds etc. which support fascinating birdlife. The present paper deals with the studies of wetland birds diversity of the Koshi Barrage area. Altogether 98 species of wetland birds belonging to 60 genera and 18 families were recorded.

Key words: Koshi Barrage,Wetland birds
\end{abstract}

\section{Introduction}

Koshi Barrage was constructed in between 1958 and 1962 across Nepal's largest river, the Sapta Koshi. The catchment area of the Sapta Koshi river is drained by seven major rivers, namely the Indrawati, Bhote Koshi, Tama Koshi, Dudh Koshi, Liku, Arun and Tamor. The Koshi Barrage was constructed by Indian government to control floods and to irrigate fields. It is located seven kilometers south of the Koshi Tappu Wildlife Reserve close to the Indo - Nepal border. Before the construction of Barrage, the Koshi River was referred to as the "Sorrow of Bihar", in India, because the Koshi River used to change its direction frequently depositing huge quantities of silt, and displacing and drowning many people every year. A reservoir has been formed over a large area near the Koshi Barrage, and the pits created after the construction of the Barrage and the embankment has provided a good habitat for residential and migratory birds. A total of 841 species of birds have been recorded in Nepal (Inskipp and Inskipp, 1991). As many as 193 species are dependent on wetlands, and among them 187 species are dependent on the terai wetlands of Nepal (Bhandari, 1998). The birds of this area have been studied by many ornithologists and naturalists (Inskipp and Inskipp, 1985; Heinen, 1986, 1987; Scott, 1989; Suwal, 1993; Baral, 2000).

\section{Materials and methods}

The population of birds was estimated from July 2002 - June 2004, every month at a regular interval simply by direct counting method. Binocular and Zoom camera were used during the study period. For identification of birds, books of Ali and Ripley (1986), Ali (1989), Ali (1990), Inskipp and Inskipp (1991), Fleming et al. (2000) and Shrestha (2000) were consulted.

\section{Results and discussion}

A total of 98 species of wetland birds belonging to 60 genera and 18 families were recorded. It comprises 41 winter visitors, 4 summer visitors, 14 occasional visitors and 39 residents (Table 1). The resident birds were observed throughout the year. Comparatively, the Koshi Barrage area is highly rich in bird diversity due to the availability of suitable habitat and food. The 


\section{T. Chhetri/ Our Nature (2006)4:91-95}

Table 1. Wetland birds around Koshi Barrage area.

\begin{tabular}{|c|c|c|c|}
\hline S.No. & Family / Scientific name & Common name & Local status \\
\hline & Family - Anatidae & & \\
\hline 1 & Anas crecca (Linnaeus) & Common Teal & Winter visitor \\
\hline 2 & Anas acuta (Linnaeus) & Pintail & , \\
\hline 3 & Anas penelope (Linnaeus) & Eurasian Wigeon & , \\
\hline 4 & Anas platyrhynchos (Linnaeus) & Mallard & , \\
\hline 5 & Anas poecilorhyncha (Linnaeus) & Spot billed Duck & , \\
\hline 6 & Anas strepera (Linnaeus) & Gadwall & , \\
\hline 7 & Anas falcata (Georgi) & Falcated Teal & , \\
\hline 8 & Anas querquedula (Linnaeus) & Garganey & , \\
\hline 9 & Anas clypeata (Linnaeus) & Shoveler & $"$ \\
\hline 10 & Sarkidiornis melanotos (Pennat) & Comb Duck & Resident \\
\hline 11 & Dendrocygna javanica (Horsfield) & Lesser whistling Teal & , \\
\hline 12 & $\begin{array}{ll}\text { Nettapus } & \text { coromandelianus } \\
\text { (Gmelin) }\end{array}$ & Cotton Teal & , \\
\hline 13 & Anser indicus (Latham) & Bar headed Goose & , \\
\hline 14 & Anser anser (Linnaeus) & Greylag Goose & , \\
\hline 15 & Tadorna tadorna (Linnaeus) & $\begin{array}{lll}\text { Common } & \text { Shelduck } & \text { (Eurasian } \\
\text { Shelduck) } & & \\
\end{array}$ & Occasional \\
\hline 16 & Tadorna ferruginea (Pallas) & Ruddy Shelduck (Brahminy Duck) & Winter visitor \\
\hline 17 & Clangula hyemalis (Linnaeus) & Long tailed Duck & Occasional \\
\hline 18 & Aythya ferina (Linnaeus) & Common Pochard & Winter visitor \\
\hline 19 & Aythya nyroca (Guldenstadt) & $\begin{array}{lll}\begin{array}{l}\text { White-eyed } \\
\text { duck) }\end{array} & \text { Pochards } & \text { (Ferruginous } \\
\end{array}$ & , \\
\hline 20 & Aythya fuligula (Linnaeus) & Tuft Pochard & , \\
\hline 21 & Netta rufina (Pallas) & Red crested Pochard & , \\
\hline 22 & Mergus merganser (Linnaeus) & Common Merganser or Goosander & , \\
\hline \multirow[t]{2}{*}{23} & Mergus albellus (Linnaeus) & Smew & Occasional \\
\hline & Family - Podicipedidae & & \\
\hline 24 & Podiceps ruficollis (Pallas) & Little Grebe & Winter visitor \\
\hline 25 & Podiceps cristatus (Linnaeus) & Great-Crested Grebe & , \\
\hline \multirow[t]{2}{*}{26} & Podiceps caspicus $($ Brehm) & Black-necked Grebe & , \\
\hline & Family -Ciconiidae & & \\
\hline 27 & Xenorhynchus asiaticus (Letham) & Blacked-necked Stork & Resident \\
\hline 28 & Leptoptilos Javanicus (Horsfield) & Lesser Adjutant Stork & , \\
\hline 29 & Ciconia nigra (Linnaeus) & Black Stork & Winter visitor \\
\hline 30 & Ciconia episcopus (Boddaert) & $\begin{array}{l}\text { White- necked Stork(Woolly necked } \\
\text { Stork) }\end{array}$ & Resident \\
\hline \multirow[t]{2}{*}{31} & Anastomus oscitans (Boddaert) & Open-billed Stork & , \\
\hline & Family -Laridae & & \\
\hline 32 & Larus brunnicephalus (Jerdon) & Brown headed Gull & Winter visitor \\
\hline 33 & Larus ichthyaetus (Pallas) & Great Black-headed Gull & , \\
\hline 34 & Sterna acuticauda (Gray) & Black-bellied Tern & Resident \\
\hline 35 & Sterna aurantia (J.E. Gray) & River Tern & , \\
\hline 36 & Sterna albifrons (Pallas) & Little Tern & Summer visitor \\
\hline 37 & Sterna hirundo (Linnaeus) & Common Tern & Winter visitor \\
\hline 38 & Chlidonias hybrida (Pallas) & Whiskered Tern & , \\
\hline
\end{tabular}




\section{T. Chhetri/ Our Nature (2006)4:91-95}

\begin{tabular}{|c|c|c|c|}
\hline & Family -Ardeidae & & \\
\hline 39 & Egretta garzetta (Linnaeus) & Little egret & Resident \\
\hline 40 & Egretta intermedia (Wagler) & Intermediate Egret & , \\
\hline 41 & Egretta alba (Linnaeus) & Large Egret & , \\
\hline 42 & Bubulcus ibis (Linnaeus) & Cattle Egret & , \\
\hline 43 & Nycticorax nycticorax (Linnaeus) & Night Heron & , \\
\hline 44 & Ardeola grayii (Sykes) & Pond Heron & , \\
\hline 45 & Ardea purpurea (Linnaeus) & Purple Heron & $\bar{l}$ \\
\hline 46 & Ardea cinerea (Linnaeus) & Grey Heron & , \\
\hline \multirow[t]{2}{*}{47} & Ixobrychus cinnamomeus (Gmelin) & Chestnut Bittern & Summer visitor \\
\hline & Family - Rallidae & & \\
\hline 48 & Gallinula chloropus(Linnaeus) & Indian Gallinule (Indian Morehen) & Winter visitor \\
\hline 49 & Porphyrio porphyrio (Linnaeus) & Purple Gallinule or Purple Moorehen & , \\
\hline 50 & Fulica atra (Linnaeus) & Coot & , \\
\hline 51 & Amaurornis phoenicurus (Pennant) & White breasted Waterhen & Resident \\
\hline \multirow[t]{2}{*}{52} & Rallus aquaticus (Blyth) & Water Rail & Winter visitor \\
\hline & Family -Threskiornithidae & & \\
\hline 53 & $\begin{array}{l}\begin{array}{l}\text { Threskiornis } \\
\text { (Letham) }\end{array} \\
\end{array}$ & White Ibis & Resident \\
\hline 54 & Pseudibis papillosa (Temminck) & Black Ibis & , \\
\hline 55 & Plegadis falcinellus (Linnaeus) & Glossy Ibis & Occasional \\
\hline \multirow[t]{2}{*}{56} & Platalea leucorodia (Linnaeus) & Eurasian Spoonbill & Winter visitor \\
\hline & Family -Phalacrocoracidae & & \\
\hline 57 & Phalacrocorax niger (Vieillot) & Little Cormorant & Resident \\
\hline 58 & Phalacrocorax carbo (Linnaeus) & Large Cormorant & Winter visitor \\
\hline \multirow[t]{2}{*}{59} & Anhinga rufa (Daudan) & Darter & Resident \\
\hline & Family -Pelecanidae & & \\
\hline \multirow[t]{2}{*}{60} & Pelicanus philippensis (Gmelin) & Spot-billed Pelican & Occasional \\
\hline & Family - Jacanidae & & \\
\hline 61 & Metopidius indicus (Latham) & Bronze-winged Jacana & Resident \\
\hline \multirow[t]{2}{*}{62} & $\begin{array}{l}\begin{array}{l}\text { Hydrophasianus } \\
\text { (Scopoli) }\end{array} \\
\end{array}$ & Pheasant-tailed Jacana & Summer visitor \\
\hline & Family -Charadriidae & & \\
\hline 63 & Tringa tetanus (Linnaeus) & Common Redshank & Winter visitor \\
\hline 64 & Tringa ochropus (Linnaeus) & Green Sandpiper & , \\
\hline 65 & Tringa Stagnatilis (Bechstein) & Marsh Sandpiper & Occasional \\
\hline 66 & Numenius arquata (Linnaeus) & Curlew & , \\
\hline 67 & Calidris alpinus (Linnaeus) & Dunlin & Winter visitor \\
\hline 68 & Calidris temminckii (Leisler) & Temminck's Stint & , \\
\hline 69 & Calidris minuta (Leisler) & Little Stint & , \\
\hline 70 & Philomachus pugnax (Linnaeus) & Ruff and Reeve & Occasional \\
\hline 71 & Gallinago gallinago (Linnaeus) & Common Snipe & Winter visitor \\
\hline 72 & Pluvialis squatarola (Linnaeus) & Grey Plover & Occasional \\
\hline 73 & Pluvialis dominica (P.L.S.Muller) & Pacific Golden Plover & Winter visitor \\
\hline 74 & $\begin{array}{ll}\begin{array}{l}\text { Charadrius } \\
\text { (Linnaeus) }\end{array} & \text { alexandrinus } \\
\end{array}$ & Kentish Plover (Snowy plover) & , \\
\hline 75 & Charadrius dubius (Scopoli) & Little ringed Plover & , \\
\hline 76 & Vanellus cinereus (Blyth) & Grey-headed Lapwing & , \\
\hline
\end{tabular}


D. T. Chhetri/ Our Nature (2006)4:91-95

\begin{tabular}{|c|c|c|c|}
\hline 77 & Vanellus vanellus (Linnaeus) & $\begin{array}{l}\text { Eurasian Lapwing or Northern } \\
\text { Lapwing }\end{array}$ & Winter visitor \\
\hline \multirow[t]{2}{*}{78} & Vanellus indicus (Boddaert) & Red wattled Lapwing & 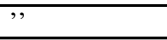 \\
\hline & Family -Recurvirostridae & & \\
\hline \multirow[t]{2}{*}{79} & Recurvirostra avosetta (Linnaeus) & Avocet & Occasional \\
\hline & Family -Burhinidae & & \\
\hline 80 & Esacus magnirostris (Vieillot) & Great Thick Knee or Stone Plover & Resident \\
\hline \multirow[t]{2}{*}{81} & Burhinus oedicnemus (Linnaeus) & Eurasian Thick Knee (Stone Curlew) & 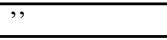 \\
\hline & Family -Glareolidae & & \\
\hline 82 & Glareola lactea $($ Temminck $)$ & Small Pratincole & Summer visitor \\
\hline \multirow[t]{2}{*}{83} & $\begin{array}{ll}\begin{array}{l}\text { Cursorius } \\
\text { (Gmelin) }\end{array} & \text { coromandelicus } \\
\end{array}$ & Indian Courser & Occasional \\
\hline & Family -Gruidae & & \\
\hline 84 & Grus grus (Linnaeus) & Common Crane & Occasional \\
\hline 85 & Grus antigone (Linneaus) & Sarus Crane & , \\
\hline \multirow[t]{2}{*}{86} & Anthropoides virgo (Linnaeus) & Demoiselle Crane & $\bar{\prime}$ \\
\hline & Family -Alcedinidae & & \\
\hline 87 & Ceryle rudis (Linnaeus) & Small Pied Kingfisher & Resident \\
\hline 88 & Ceryle lugubris (Temminck) & $\begin{array}{l}\text { Large Pied Kingfisher or Crested } \\
\text { Kingfisher }\end{array}$ & , \\
\hline 89 & Alcedo meninting (Horsfield) & Blue eared Kingfisher & , \\
\hline 90 & Alcedo atthis (Linnaeus) & Eurasian Kingfisher & , \\
\hline 91 & Halcyon coromanda (Letham) & Ruddy Kingfisher & , \\
\hline 92 & Halcyon smyrnensis (Linnaeus) & White breasted Kingfisher & , \\
\hline \multirow[t]{2}{*}{93} & Pelargopsis capensis (Linnaeus) & Stork-billed Kingfisher & , \\
\hline & Family-Rostratulidae & & \\
\hline \multirow[t]{2}{*}{94} & $\begin{array}{ll}\begin{array}{l}\text { Rostratula } \\
\text { (Linnaeus) }\end{array} & \text { benghalensis } \\
\end{array}$ & Painted Snipe & Resident \\
\hline & Family - Motacillidae & & \\
\hline 95 & Motacilla flava (Linnaeus) & Yellow Wagtail &, \\
\hline 96 & Motacilla alba (Linnaeus) & Pied Wagtail(White Wagtail) & , \\
\hline 97 & Motacilla citreola (Pallas) & Yellow-headed Wagtail & , \\
\hline 98 & $\begin{array}{ll}\begin{array}{l}\text { Motacilla } \\
\text { (Gmelin) }\end{array} & \text { maderaspatensis } \\
\end{array}$ & Large Pied Wagtail & Resident \\
\hline
\end{tabular}

present study recorded $11.65 \%, 50.77 \%$, and $52.41 \%$ species of total birds of Nepal, total wetlands' birds of Nepal and total terai wetlands' birds of Nepal respectively. The winter visitor species were recorded maximum during the study period and summer visitors were minimum. Some species like Common Crane, Long tailed Duck, Spot-billed Pellican etc. were observed occasionally. The maximum winter visitor species occurred in January followed by February and March. The pintail was most abundant followed by the Common Teal, Lesser Whistling Teal, Gadwall and Cotton Teals respectively. The number of birds recorded during this study period was compared with earlier records ( Scott, 1989), and it was found in decreasing trend in the abundance of Pintail, Garganey, Lesser Whistling Teal, Ruddy Shelduck etc. There may be several reasons for the decrement of abundance of birds species, 


\section{T. Chhetri/ Our Nature (2006)4:91-95}

one of them may be that birds have changed their migration route, although this reason unlikely. The other reasons are hunting, poaching, trapping in the migration route, and in the breeding areas. Besides these, possible reasons nowadays the human disturbances, increased food shortage and deposition of silt around the Koshi Barrage area are main.

\section{References}

Ali, S.1989.The Birds of Sikkim. Oxford University Press.

Ali, S.1990.The Book of Indian Birds. Oxford University Press

Ali, S. and S.D. Ripley. 1986. Handbook of the Birds of India andPakistan, Vol. V. Oxford University Press, London.

Baral, H.S. 2000. Birds of Koshi. Bird conservation Nepal, Department of National Parks and Wildlife Conservation, Kathmandu, Nepal.
Bhandari, B.1998. Avifauna. In : An Inventory of Nepal's Terai Wetlands. IUCN, Nepal. pp. 48-49.

Fleming, R.L., R.J. Fleming, Jr. and L.S. Bangdel. 2000. Birds of Nepal with reference to Kashmir and Sikkim. First Adarsh Impression, Gaurav Offset Delhi.

Heinen, J.T. 1986. Rare and New Birds Record for Koshi Tappu and Koshi Barrage for winter and spring. J. Nat. Hist. Mus.10: 23-30.

Heinen J.T. 1987. New Seasonal and Range Records and Status Updates for Birds in Koshi Tappu Wildlife Reserve and the Koshi Barrage, Nepal. J. Nat. Hist. Mus.11: 41-50.

Inskipp, C. and J. Inskipp. 1985. A Guide to the Birds of Nepal. Christopher Helm. London.

Inskipp, C. and T. Inskipp. 1991. A Guide to the Birds of Nepal. Christopher Helm.London.

Scott, D.A. 1989. A Directory of Asian Wetlands. IUCNThe World Conservation Union, Gland, Switzerland.

Shrestha, T.K. 2000. Birds of Nepal. Mrs. Bimala Shrestha, Kathmandu, Nepal.

Suwal, R.N.1993. Koshi Tappu Wildlife Reserve: Conservation Issues and Management Measures. A Survey Report submitted to IUCN, Nepal. 